\title{
Synthesis of Planar Filters Using Defected Ground Structure Miniaturized Hairpin Resonators
}

\author{
Marin Nedelchev \\ Radiocommunication and Videotechnologies Dept. \\ Faculty of Telecommunications \\ Technical University Sofia \\ Sofia, Bulgaria \\ mnedelchev@tu-sofia.bg
}

\author{
Aleksandar Kolev \\ Radiocommunication and Videotechnologies Dept. \\ Faculty of Telecommunications \\ Technical University Sofia \\ Sofia, Bulgaria \\ alex.n.kolev@gmail.com
}

\begin{abstract}
This paper presents an effective technique to suppress the spurious passbands in planar filters by using defected ground structure (DGS) resonators etched in the ground plane. The proposed miniaturized hairpin DGS slot resonator is researched in terms of its resonance frequency, corresponding coupling topologies, and filter design. The resonator and the topologies of coupled DGS resonators are simulated in a fullwave electromagnetic (EM) simulator. Using a curve-fitting technique, useful design formulas are proposed for filter synthesis. Using the synthesis procedure, a 3rd order filter design is simulated, manufactured and measured. The insertion loss of $-3 \mathrm{~dB}$ in the passband of $280 \mathrm{MHz}$ is observed, while the suppression of the spurious passbands up to $12 \mathrm{GHz}$ is more than $24 \mathrm{~dB}$.
\end{abstract}

Keywords-microstrip; defected ground resonator; coupling coefficient; slot line filter deisgn

\section{INTRODUCTION}

Bandpass filters used in modern microwave communication systems have to comply with very strict requirements about their performance, size and volume. The manufacturing process of such filters must be technologically easy to produce and adjust. Many compact microstrip resonators are reported in [110]. The introduction of etched slots in the ground plane of a microstrip line adds degrees of freedom in the design and synthesis of microstrip filters. These are also known as defected ground structures (DGS). They can be periodic or non-periodic disturbances in the ground plane of the microstrip line. Their shape can be adopted from microstrip resonators [1] and appears to be dual to them. This encourages the development of new topologies of planar filters with special specifications. The use of half wave square open loop and miniaturized hairpin resonators as DGS is researched in-depth in [5-8]. A combination of microstrip resonators and DGS resonators are used in [8] as building elements of planar filters. Both applications of slot resonators are a promising way of adding additional degrees of freedom in filter design. The use of DGS resonators can solve a serious problem in filter design the small gap that is required between coupled lines in order to achieve strong coupling. One of the problems encountered in the design of wideband and ultra-wideband filters is the physical realization of very small gaps between coupled resonators. The manufacturing tolerances and the precision of the manufacturing process associated with realization of small gaps affect the frequency response of the designed filter. By utilizing different shaped slots in the ground plane of the microstrip line - DGS [1-3, 5-8], it is possible to enhance the coupling coefficient.

This paper researches miniaturized hairpin DGS resonator and the coupling structures formed by placing two resonators close to each other. The resonance frequency of the DGS resonator is investigated and a design formula is proposed. The main topologies of coupled DGS resonators are researched and based on the simulation results, simple formulas are proposed using a curve fitting technique. A three-resonator filter is synthesized, simulated, produced and measured in order to verify the design equations. There is a good agreement between theoretical, simulated and measured results.

\section{MINIATURIZED HAIRPIN DGS RESONATOR}

All the simulations and design procedures in this paper are performed in Ansys Electromagnetics Suite v.17.2 for a FR-4 dielectric substrate with $\varepsilon_{r}=4.4,1.5 \mathrm{~mm}$ height and loss tangent $\operatorname{tg} \delta=0.02$. The miniaturized hairpin microstrip resonator is proposed and its synthesis method is described in [1, 3]. The resonator consists of a main slot line loaded with two parallelcoupled slots with a small gap between them. The etched resonator is symmetrical around the axis and the open end is in the middle of the main line. The magnetic field is concentrated in the coupled lines and the electric field is at its maximum near the open end of the resonator. Based on the field distribution there are three main coupling topologies that can be realized, namely electric, magnetic and mixed coupling. The occupied area of a miniaturized hairpin DGS resonator is less than the conventional hairpin or slow wave resonators. This makes the miniaturized hairpin DGS resonator suitable for devices working in the lower microwave bands, where the physical dimensions of the transmission lines are relatively large and miniaturization is not possible. Another advantage of the DGS resonator is its rectangular form, which allows its usage in the design of canonical and pseudo-elliptic filters with cross coupling. 
Coupled slot lines can be used for altering the resonant frequency of the resonator. The dimensions of the resonator tuned to central frequency $f_{0}=2.4 \mathrm{Gzh}$ are shown in Figure 1 . The main slot's width is $w=2.8 \mathrm{~mm}$ which equals the width of a $50 \Omega$ microstrip line for the used substrate. Figure 2 shows the dependence between the length of the coupled lines $p$ and the first resonant frequency of the resonator, obtained with fullwave electromagnetic simulations. Following the simulation results, the resonance frequency of the DGS resonator is easily tuned by adding or removing metal to the ground plane. Using a curve fitting technique, a useful expression is derived for the length of the coupled arms:

$$
p=103.8 e^{-0.9858 f_{0}[\mathrm{GHz}]},[\mathrm{mm}]
$$

The accuracy of (1) compared to the electromagnetic simulations is better than $2.5 \%$ and prevents errors caused by a wrong read of the graphic results shown in Figure 2.

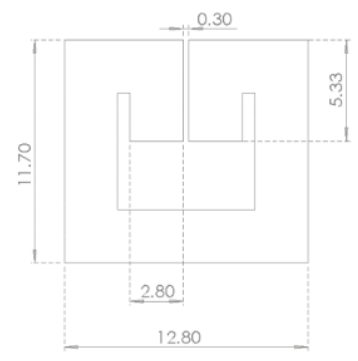

Fig. 1. Dimensions of the DGS resonator tuned to $f_{0}=2.4 \mathrm{Gzh}$

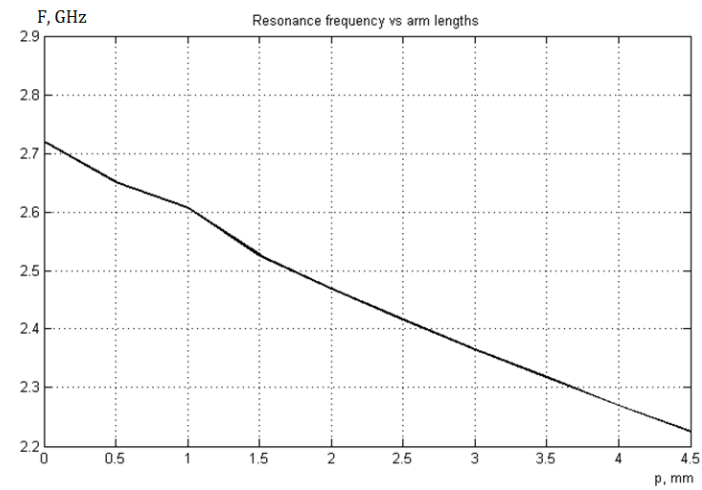

Fig. 2. Dependence of the resonant frequency of the coupled lines length

\section{COUPLING COEFFICIENTS AND EXTERNAL QUALITY FACTOR SIMULATIONS}

As described in [2], the coupling coefficient for synchronously tuned resonators can be calculated by finding the eigenfrequencies of even $\left(f_{e}\right)$ and odd $(f)_{o}$ mode associated with coupling between a pair overcoupled resonators.

$$
k=\frac{f_{e}^{2}-f_{o}^{2}}{f_{e}^{2}+f_{o}^{2}}
$$

A finite element method (FEM) based fullwave EM simulator is used to identify the resonance frequencies in the response [2]. Figure 3 shows the coupling topologies used to evaluate the coupling coefficients and the external quality factor. The miniaturized hairpin slot resonators are dual to the miniaturized hairpin resonators and the electromagnetic field is inversely distributed in it. The magnetic field has a maximum value in the connection point of the coupled lines with the transmission line and the electric field has a maximum value in the center of the main slot line.

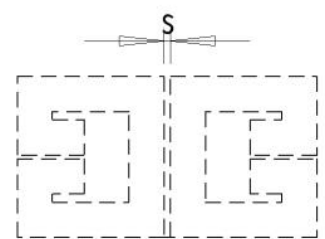

(a)

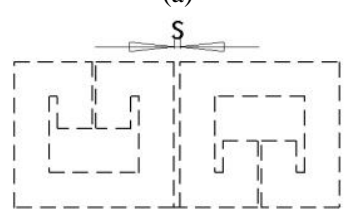

(c)

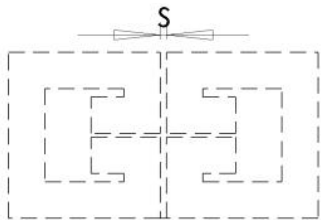

(b)

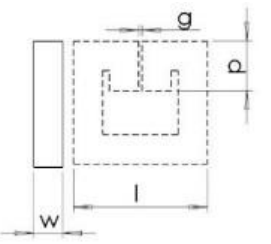

(d)
Fig. 3. Coupling topologies of miniaturized hairpin DGS resonators: (a) electric, (b) magnetic, (c) mixed, (d) external quality factor

There are three main types of coupling topologies electric, magnetic and mixed. The electric coupling is shown on Figure 3(a), where the electric field has a maximum and dominates over the magnetic field. In this configuration the sign of the coupling coefficient is negative and can be used for cross coupled filters. On Figure 3(b) the magnetic coupling is shown. In this configuration, the magnetic field is dominant and the sign of the coupling coefficient is positive. The mixed coupling is shown on Figure 3(c). In this case neither the electric nor the magnetic field is dominant. When a microstrip filter is being synthesized, an important point is the determination of the gap between the coupled resonators with respect to the value of the coupling coefficient calculated from the approximation. Three main approaches exist to determine the separation between the resonators: analytic formulas [2], approximate formulas from curve fitting [8] and extraction from EM simulations [2, 3]. Using (2) and EM simulation of the coupling structures, which are weekly coupled to a $50 \Omega$ microstrip feed line the coupling coefficients are extracted. Figures 4-6 show the coupling coefficients for the three types of coupling as a function of the distance between the resonators $s$. Using a curve fitting method, the following dependences are derived for design purposes:

For electric coupling :

$s_{e}=6.88 e^{-40.36 M_{e}}$

For magnetic coupling:

$s_{m}=12.36 e^{-18.45 M_{m}}$

For mixed coupling:

$S_{\text {mix }}=13.05 e^{-28.73 M_{\text {mix }}}$ 


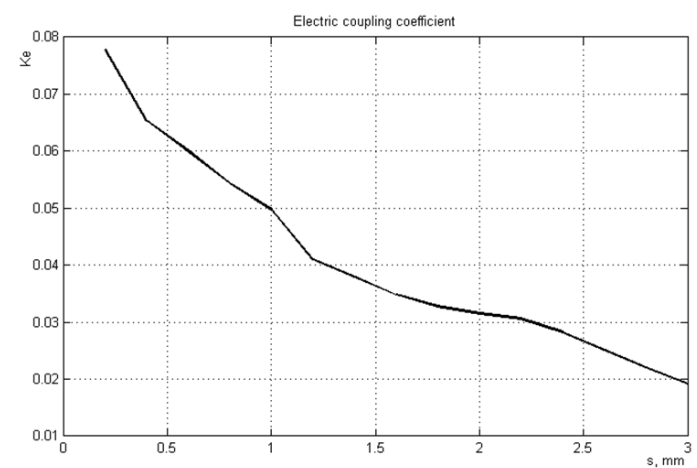

Fig. 4. Coupling coefficient as function of the separation between the resonators for the electric coupling

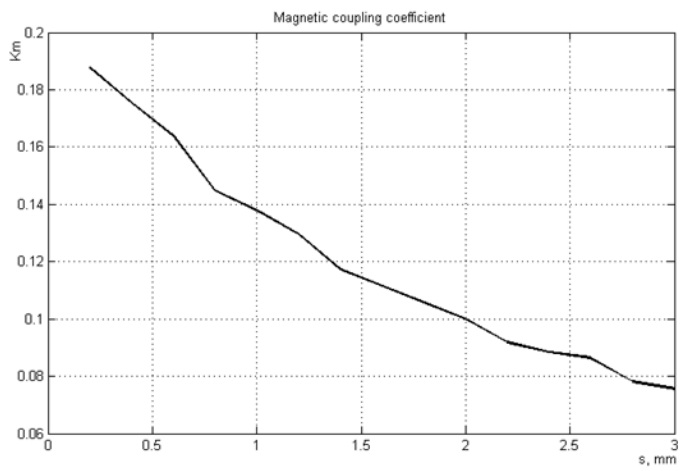

Fig. 5. Coupling coefficeint as function of the separation between the resonators for the magnetic coupling

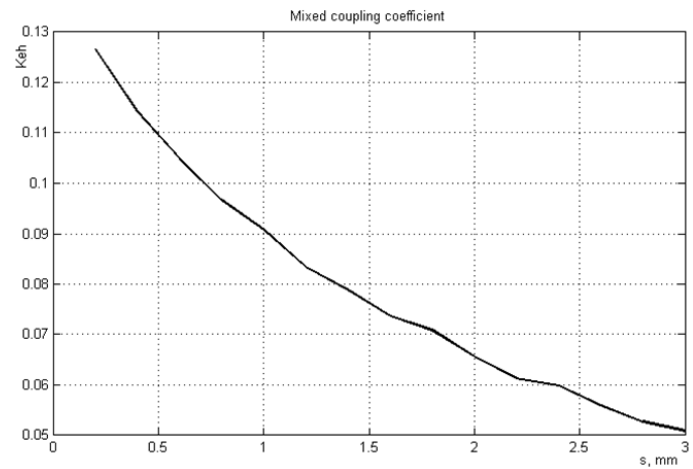

Fig. 6. Dependance of thecoupling coefficient as a function of the separation between the resonators for the mixed coupling

By using (3)-(5) it is easy to compute the separation between the coupled lines with accuracy better than 5\% compared to the EM simulations. The external quality factor defines the position of the input/output lines. In the case of miniaturized hairpin slot resonators, the external quality factor is realized by a $50 \Omega$ microstrip line on the top layer of the substrate (Figure 3(d)). The dependence of the external quality factor as a function of the linear position of the microstrip line is shown on Figure 7. The input/output microstrip line influences the resonance frequency of the slot resonator and shits it towards lower frequencies. This effect must be compensated while the filter is being designed by reducing the length of the coupled lines of the input/output resonators. Because the feed line is placed on the top layer of the substrate, input/output lines can overlap the slot resonators. The external quality factor changes its value at a very high rate when the microstrip line is placed near the DGS resonator.

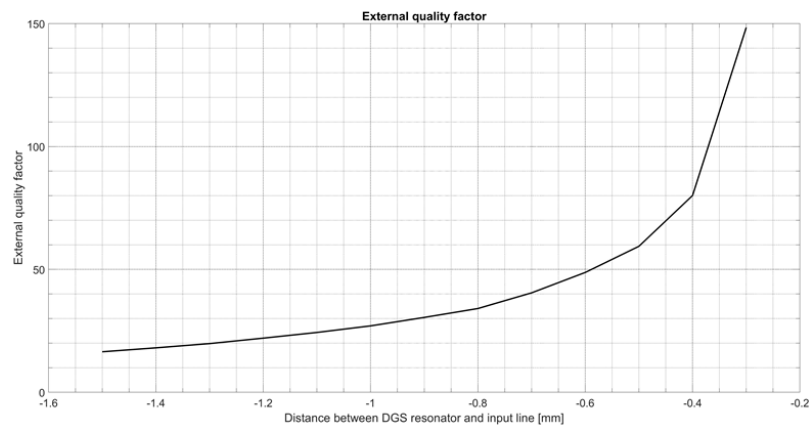

Fig. 7. Dependance of the external coupling as a function of the position of the input/output microtrip line

\section{BANDPASS FILTER SYNTHESIS, SIMULATIONS AND MEASUREMENTS}

The proposed topologies of coupled resonators and the formulas for the coupling coefficients can be verified by designing a three-resonator Chebyshev filter. The required coupling coefficients must be computed by using the standard technique described in [2]:

$$
M_{n, n+1}=\frac{F B W}{\sqrt{g_{n} g_{n+1}}}
$$

where $F B W$ is the fractional bandwidth and $g_{n}, n=0,1,2,3$ are the values of the elements of the lowpass filter prototype. Different sources of precomputed values for the elements for different pass band ripple can be found. The current filter is designed for center frequency $f_{0}=2400 \mathrm{MHz}$, bandwidth $\Delta f=200 \mathrm{MHz}$ and return loss in the pass band $R L=-20 \mathrm{~dB}$. The values for the coupling coefficients are $M_{12}=M_{23}=0.086$ and the external quality factor is $Q_{e}=25.92$. The gaps between the resonators are $s_{12}=s_{23}=1.13 \mathrm{~mm}$ and the overlapping between the input/output line and slot resonator is $d=-1.25 \mathrm{~mm}$. Ansys Electromagnetics suite v.17.2 is used to simulate the filter in its planar simulator. The topology of the simulated filter is shown on Figure 8 and the results are shown on Figures 10 and 11. The simulated filter has a bandwidth of $280 \mathrm{MHz}$ and the coupling between the resonators appears to be stronger than designed. Due to the effect of the input/output lines on the first and third resonators frequency response the length of the coupled lines has to be reduced by $0.6 \mathrm{~mm}$ in order to achieve the desired frequency. From Figure 10 it is seen that the filter has no spurious pass band response up to $12 \mathrm{GHz}$. The minimum suppression of the out of bandpass is $-23 \mathrm{~dB}$, while the maximum suppression is below $-45 \mathrm{~dB}$. The designed filter was produced using standard PCB manufacturing technology on a FR-4 substrate with $1.5 \mathrm{~mm}$ thickness and $0.035 \mathrm{~mm}$ copper foil (Figure 11). On Figure 12 the simulated and measured frequency responses for the $s_{11}$ and $s_{21}$ in narrowband are both shown. The measurements were performed on a 2 port network analyzer PNA-X N5242B. There is good agreement between the simulated and the measured results. 


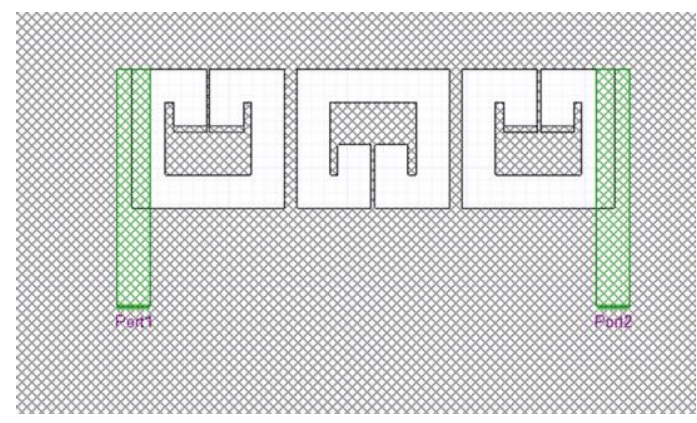

Fig. 8. Topology of the synthesized three resonator Chebyshev filter

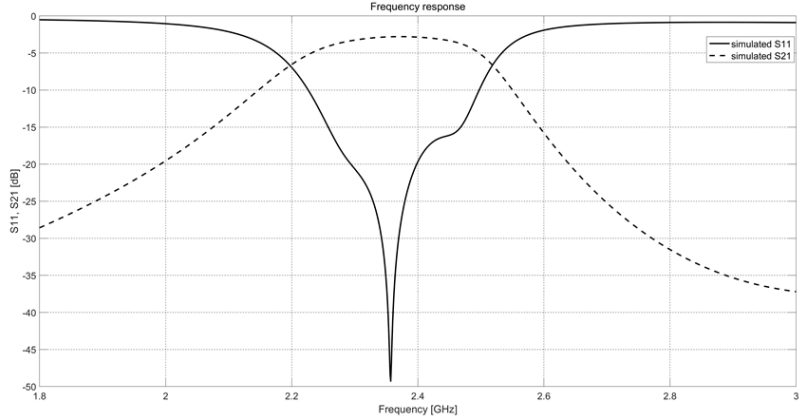

Fig. 9. Simulated narrowband frequency response of the synthesized filter.

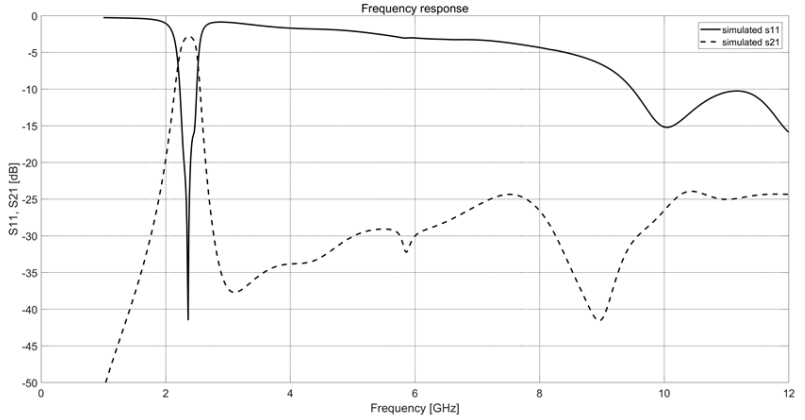

Fig. 10. Simulated wideband frequency response of the synthesized filter

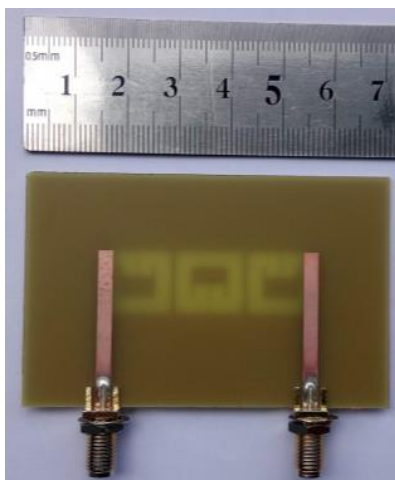

(a)

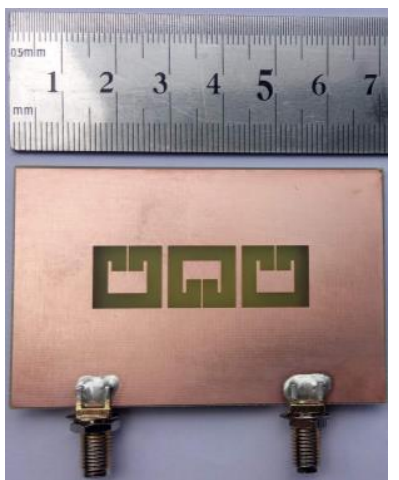

(b)
Fig. 11. Fabricated PCB of the synthesized three resonator Chebyshev filter: (a) top layer, (b) bottom layer

The measured bandwidth of the filter is $287 \mathrm{MHz}$, while the insertion loss in the passband is $-2.79 \mathrm{~dB}$. The maximum value of the reflection coefficient in the passband is $-20 \mathrm{~dB}$, which corresponds to $0.1 \mathrm{~dB}$ ripple of the transmission coefficient. The measured center frequency of the filter is $2.35 \mathrm{GHz}$. Figure 13 shows the wideband frequency response of the simulated and measured filter. The measured suppression of the spurious passbands is lower than $-24 \mathrm{~dB}$ as predicted in the filter simulations.

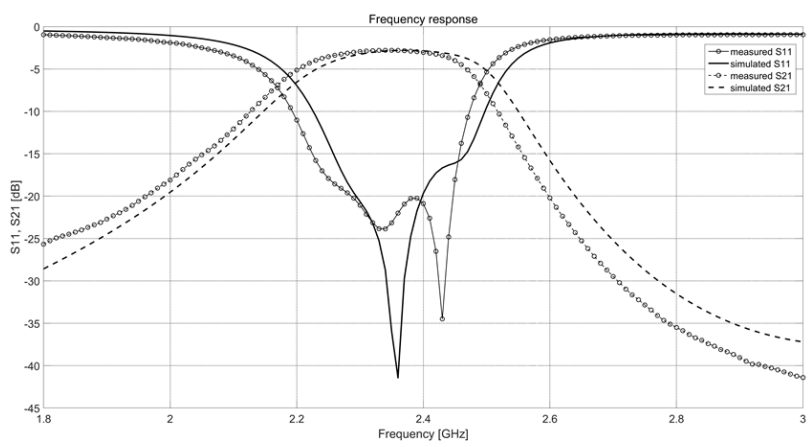

Fig. 12. Measured and simulated narrowband freqeuncy response of the synthesized filter

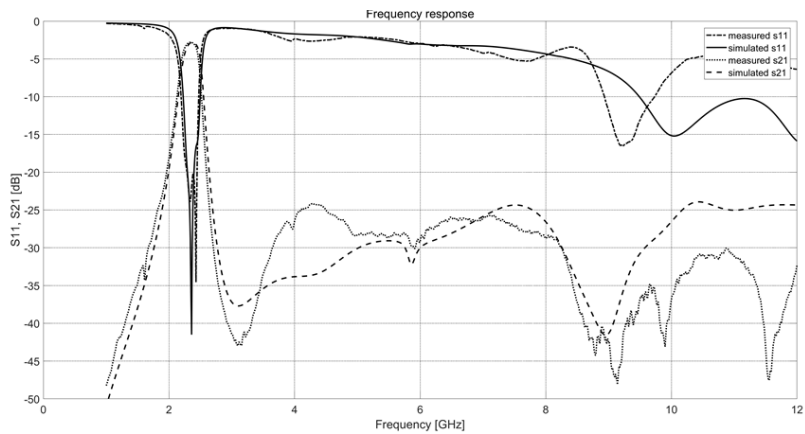

Fig. 13. Measured and simulated wideband freqeuncy response of the synthesized filter

\section{CONCLUSION}

A design of a DGS resonator and its corresponding coupling structures are presented in this paper. Topologies of coupled DGS resonators are researched and the use of curve fitting technique designed formulas is proposed. A three resonator filter is synthesized, simulated and produced in order to verify the design equations. A good agreement between the simulated, theoretical and measured results is observed. The proposed DGS resonators can be used in the design of microstrip filters in the ISM band on $2.4 \mathrm{GHz}$.

\section{ACKNOWLEDGEMENT}

This work has been supported by the research grant No 182ПД0011-07 with the Scientific Research Center Technical University of Sofia.

\section{REFERENCES}

[1] M. Makimoto, S. Yamashita, Microwave Resonators and Filters for Wireless Communication, Theory Design and Applications, Springer, 2001

[2] J. Hong, M. J Lancaster, Microstrip Filters for RF/Microwave Applications, John Wiley and Sons, 2001 
[3] R. Garg, I. Bahl, M. Bozzi, Microstrip Lines and Slotlines, Artech House 2013

[4] H. J. Chen, T. H. Huang, C. S. Chang, L. S. Chen, N. F. Wang, Y. H. Wang, M. P. Houng, "A Novel Cross-Shape DGS Applied to Design Ultra-Wide Stopband Low-Pass Filters," IEEE Microwave and Wireless Component Letters, Vol. 16, No. 5, pp. 252-254, 2006

[5] A. Abdel-Rahman, A. R. Ali, S. Amari, A. S. Omar, "Compact bandpass filters using defected ground structure (DGS) coupled resonators", 2005 IEEE MTT-S International Microwave Symposium Digest, Long Beach, USA, June 12-17, 2005

[6] P. Singh, R. Tomar, P. Bhartia, "The use of defected ground structures in designing microstrip filters with enhanced performance characteristics", Procedia Technology, Vol. 17, pp. 58-64, 2014

[7] A. R. Ali, A. Abdel-Rahman, S. Amari, A. S. Omar, "Direct and crosscoupled resonator filters using defected ground structure (DGS) resonators". 2005 European Microwave Conference, Paris, France, October 4-6, 2005

[8] P. Vagner, M. Kasal, "A Novel Bandpass Filter Using a Combination of Open-Loop Defected Ground Structure and Half-Wavelength Microstrip Resonators", Radioengineering, Vol. 19, No. 3, pp. 392-396, 2010

[9] S. K. Parui, S. Das, "A New Defected Ground Structure for Different Microstrip Circuit Applications", Radioengineering Vol. 16, No 1, pp. 16-16, 2007

[10] D. J. Woo, T. K. Lee, "Suppression of Harmonics in Wilkinson Power Divider Using Dual-Band Rejection by Asymmetric DGS", IEEE Transactions on Microwave Theory and Techniques, Vol. 53, No. 6, pp. 2139-2144, 2005 